\title{
High Ion Temperature Plasmas using an ICRF Wall-Conditioning Technique in the Large Helical Device
}

\author{
Hiromi TAKAHASHI, Masaki OSAKABE, Sadayoshi MURAKAMI ${ }^{1)}$, Kenichi NAGAOKA, \\ Haruhisa NAKANO, Yasuhiko TAKEIRI, Tetsuo SEKI, Kenji SAITO, Hiroshi KASAHARA, \\ Shuji KAMIO, Takashi MUTOH and the LHD Experiment Group \\ National Institute for Fusion Science, 322-6 Oroshi-cho, Toki 509-5292, Japan \\ ${ }^{1)}$ Department of Nuclear Engineering, Kyoto University, Kyoto 606-8501, Japan
}

(Received 16 December 2013 / Accepted 8 March 2014)

\begin{abstract}
A new effective wall-conditioning technique was proposed for realizing high ion temperature $\left(T_{\mathrm{i}}\right)$ plasmas in the Large Helical Device using the ion cyclotron range of frequency (ICRF) wave under the established magnetic confinement field. A series of ICRF heating discharges using He as the working gas was conducted ahead of the high- $T_{\mathrm{i}}$ plasma discharges. After sufficient repetitive wall-conditioning discharges, we observed a decrease in the line-averaged electron density, the formation of a peaked electron density profile, a reduction in the $\mathrm{H} \alpha$ emission, and an increase in the central $T_{\mathrm{i}}$. The results suggest that the stored hydrogen inside the vacuum vessel structures, such as the first wall, the diverter, and other components, sputtered out owing to the He plasmas of the ICRF wall-conditioning discharges and the hydrogen recycling was decreased. Consequently, the ion heating power of NBI increased in the plasma core region, leading to higher central $T_{\mathrm{i}}$.
\end{abstract}

(C) 2014 The Japan Society of Plasma Science and Nuclear Fusion Research

Keywords: wall conditioning, recycling, plasma wall interaction, ICRF, high temperature, Large Helical Device

DOI: $10.1585 /$ prr.9.1402050

\section{Introduction}

The wall conditions substantially affect the plasma performance in the toroidal system [1-7]. In the Large Helical Device (LHD), high-performance plasmas such as super-dense core plasmas and high ion temperature $\left(T_{\mathrm{i}}\right)$ plasmas have been realized owing to peripheral particle control techniques such as the local island diverter, glow discharges, and titanium gettering [8-10]. Recently, high$T_{\mathrm{i}}$ plasmas, which were produced using high-power NBIs, were obtained using a series of wall-conditioning discharges of the $38-\mathrm{MHz}$ ion cyclotron range of frequency (ICRF) wave ahead the high- $T_{\mathrm{i}}$ plasma discharges [11]. In typical high- $T_{\text {i }}$ plasmas in the LHD, hollow electron density $\left(n_{\mathrm{e}}\right)$ profiles have been observed. In contrast, a peaked $n_{\mathrm{e}}$ profile and higher $T_{\mathrm{i}}$ were obtained after the ICRF wallconditioning discharges.

In this study, we discuss the effect of the ICRF wallconditioning on the production of high- $T_{\mathrm{i}}$ plasmas in the LHD. First, the new wall-conditioning technique using the ICRF wave is introduced. Next, the production of the high$T_{\mathrm{i}}$ plasmas using the ICRF wall-conditioning technique is presented. The effect of the ICRF discharges on the reduction of hydrogen recycling from the vacuum vessel components is discussed. Finally, the results are summarized.

\section{ICRF Wall-Conditioning under the Established Confinement Magnetic Field}

The LHD is the largest heliotron device in the world with a major radius of $3.9 \mathrm{~m}$, a minor radius of $0.6 \mathrm{~m}$, a pole number of 2 , and a toroidal period of 10 [12-14]. The heliotron configurations are produced by a set of helical

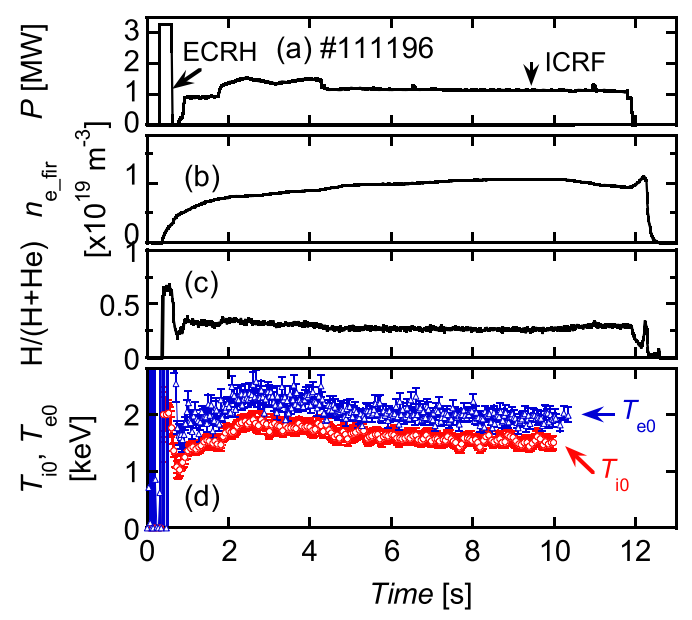

Fig. 1 Typical time evolution of (a) the heating power of the ICRF wave and ECRH, (b) the line-averaged electron density $n_{\mathrm{e}_{-} \text {fir }}$, (c) the $\mathrm{H} /(\mathrm{H}+\mathrm{He}$ ) value, and (d) the central temperature of ion $T_{\mathrm{i} 0}$ and electron $T_{\mathrm{e} 0}$. 

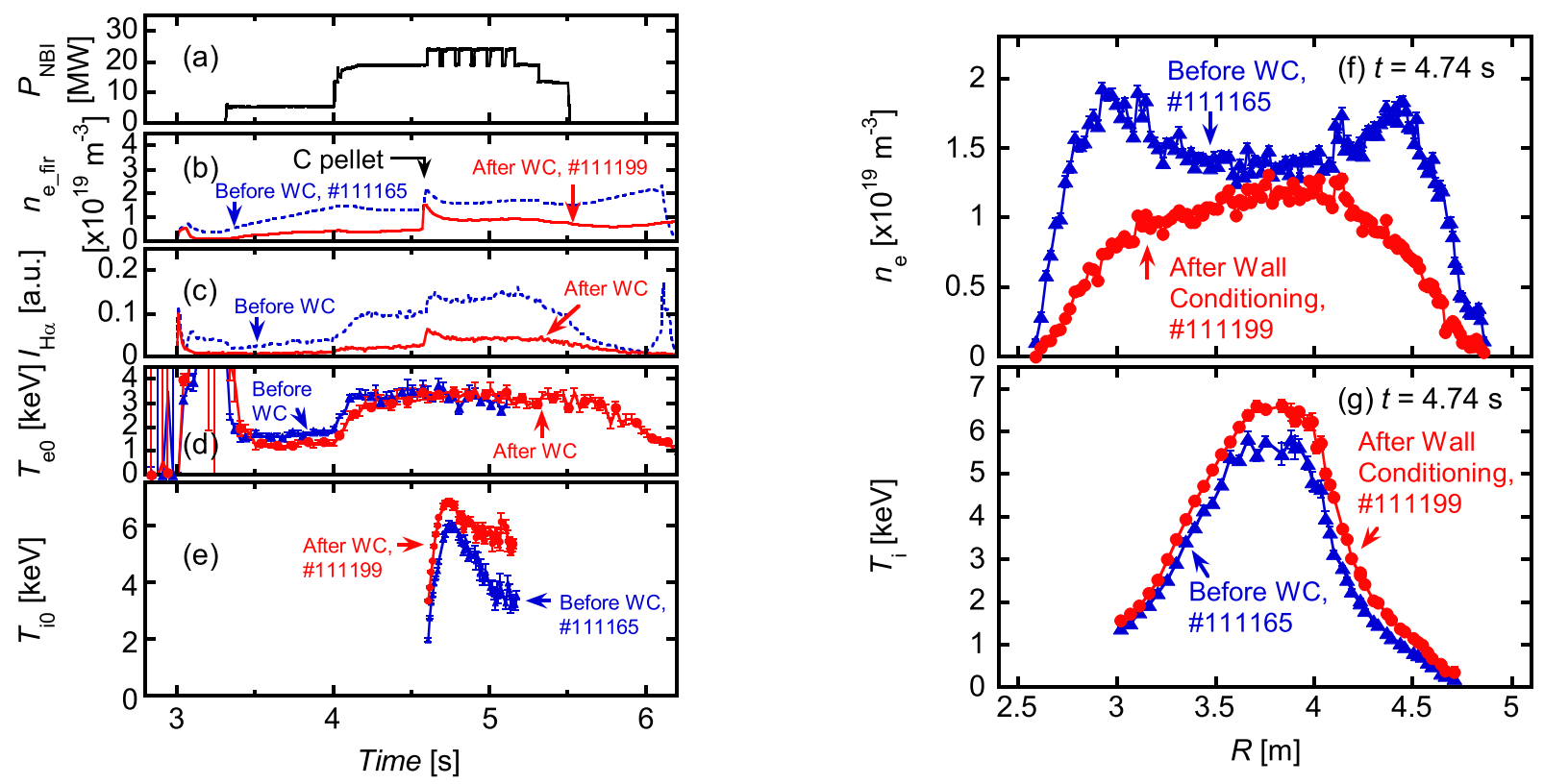

Fig. 2 Time evolution of (a) the NBI port through power $P_{\mathrm{NBI}}$, (b) $n_{\mathrm{e} \_ \text {fir }}$, (c) $\mathrm{H} \alpha$ emission intensity $I_{\mathrm{H} \alpha}$, (d) $T_{\mathrm{e} 0}$, (e) $T_{\mathrm{i} 0}$, the radial profiles of (f) $n_{\mathrm{e}}$, and (g) $T_{\mathrm{i}}$ at $t=4.74 \mathrm{~s}$ for the high- $T_{\mathrm{i}}$ discharges just before (\#111165) and after (\#111199) the ICRF wall-conditioning.

coils and three sets of poloidal field coils, which are all superconducting magnets. Therefore, the steady-state formation of magnetic configuration and plasma sustainment are realized. The magnetic field on the axis is $\sim 2.9 \mathrm{~T}$.

The new wall-conditioning discharges using the ICRF wave were conducted ahead of the high- $T_{\mathrm{i}}$ discharges. Figure 1 shows the typical time evolution of (a) the heating power of the ICRF wave and electron-cyclotron resonance heating $(\mathrm{ECRH}),(\mathrm{b})$ the line-averaged electron density $n_{\mathrm{e}_{-} \text {fir }}$, (c) the $\mathrm{H} /(\mathrm{H}+\mathrm{He})$ value [15], and (d) the central temperature of ion $T_{\mathrm{i} 0}$ and electron $T_{\mathrm{e} 0}$. The discharge was conducted using $\mathrm{He}$ as the working gas for the ICRF heating under the established confinement magnetic field of $R_{\mathrm{ax}}=3.6 \mathrm{~m},\left|B_{\mathrm{t}}\right|=2.75 \mathrm{~T}$. The plasma was ignited using $3 \mathrm{MW}$ ECRH and was steadily sustained with $n_{\mathrm{e}}$ of $0.7-1 \times 10^{19} \mathrm{~m}^{-3}$ using 38-MHz ICRF heating only for $12 \mathrm{~s}$. Low value of an $\mathrm{H} /(\mathrm{H}+\mathrm{He})$ is suitable for the effective hydrogen minority heating of the ICRF wave. Although the plasma was maintained during the ICRF wave injection, $T_{\mathrm{i} 0}$ and $T_{\mathrm{e} 0}$ are shown up to $10 \mathrm{~s}$ owing to data acquisition limitations. The preferred conditions for high- $T_{\mathrm{i}}$ plasmas are realized after several tens ICRF wall-conditioning discharges.

\section{Effect of the ICRF Wall Condition- ing on the High- $T_{\mathrm{i}}$ plasma Perfor- mânce}

Figure 2 shows the time evolution of (a) the NBI port through power $P_{\mathrm{NBI}}$, (b) $n_{\mathrm{e}-\text { fir }}$, (c) $\mathrm{H} \alpha$ emission intensity $I_{\mathrm{H} \alpha}$, (d) $T_{\mathrm{e} 0}$, (e) $T_{\mathrm{i} 0}$, (f) radial profiles of $n_{\mathrm{e}}$, and (g) $T_{\mathrm{i}}$ at $t=4.74 \mathrm{~s}$ for the high $-T_{\mathrm{i}}$ discharges just before (\#111165) and after (\#111199) the ICRF wall conditioning. Between the two discharges, 30 ICRF conditioning discharges were conducted. In the LHD, high- $T_{\mathrm{i}}$ plasmas are typically produced using high-power NBI and carbon pellets $[10,11]$. A column-shaped carbon pellet $(\phi 1.0 \mathrm{~mm} \times l 1.0 \mathrm{~mm})$ was injected at $t=4.52 \mathrm{~s}$ and $n_{\mathrm{e}-f i r}$ increased sharply. In the density decay phase, $T_{\mathrm{i} 0}$ increased until $\sim 4.75 \mathrm{~s}$ and then gradually decreased. In the plasma, just after the ICRF conditioning discharges, we observed a decrease in the averaged density, high $T_{\mathrm{i}}$, a clear reduction in the $\mathrm{H} \alpha$ signal, and a change in the $n_{\mathrm{e}}$ profile from hollow to peaked. The reduction in $\mathrm{H} \alpha$ represents a decrease in hydrogen recycling from in-vessel components. The change in the $n_{\mathrm{e}}$ profile is due to the decrease in hydrogen recycling. Consequently, the NBI absorption power per ion increased in the plasma core region leading to the high- $T_{\mathrm{i}}$ plasma [11].

Figure 2 also shows the wall-conditioning effect for the different methods. Before starting the experiment, twohour He glow discharge and 4-hour titanium gettering were performed. Discharge \#111165 was obtained early in the day (fifth shot), and thus was considered to have substantially benefited from the wall conditionings. Comparing the attained $T_{\mathrm{i} 0}$ in the discharges, just after the different wall-conditioning methods, the ICRF wall conditioning in the established magnetic field was found more effective than the conventional wall-conditioning technique for realizing high- $T_{\mathrm{i}}$ plasmas.

\section{Discussion}

Figure 3 shows the time trend of (a) the hydrogen pressure $P_{\mathrm{H} 2}$ inside the LHD, (b) $T_{\mathrm{i} 0}$ at $t=4.74 \mathrm{~s}$ and the integration of the input ICRF energy $W_{\text {ICRF }}$ during a typical 

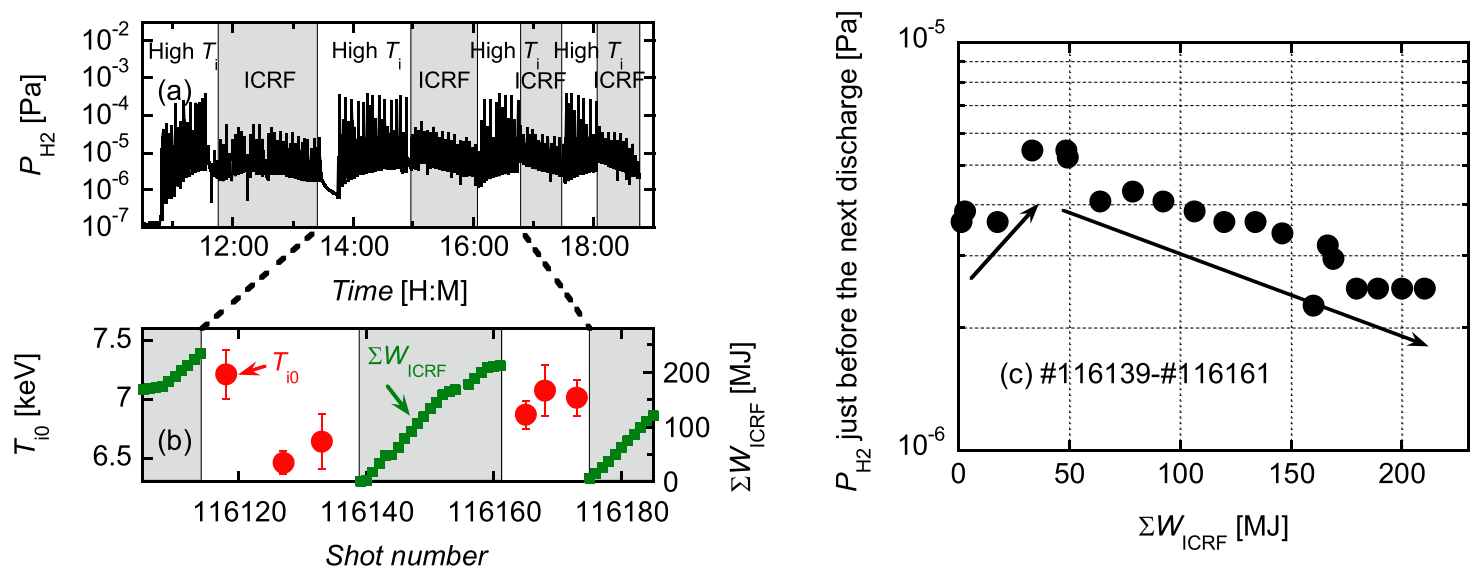

Fig. 3 Time trend of (a) the hydrogen pressure $P_{\mathrm{H} 2}$ inside the LHD, (b) $T_{\mathrm{i} 0}$ at $t=4.74 \mathrm{~s}$ and the integration of the input ICRF energy $W_{\mathrm{ICRF}}$ in an LHD experiment day and (c) the relation between $\Sigma W_{\mathrm{ICRF}}$ and $P_{\mathrm{H} 2}$ just before the next ICRF discharge.
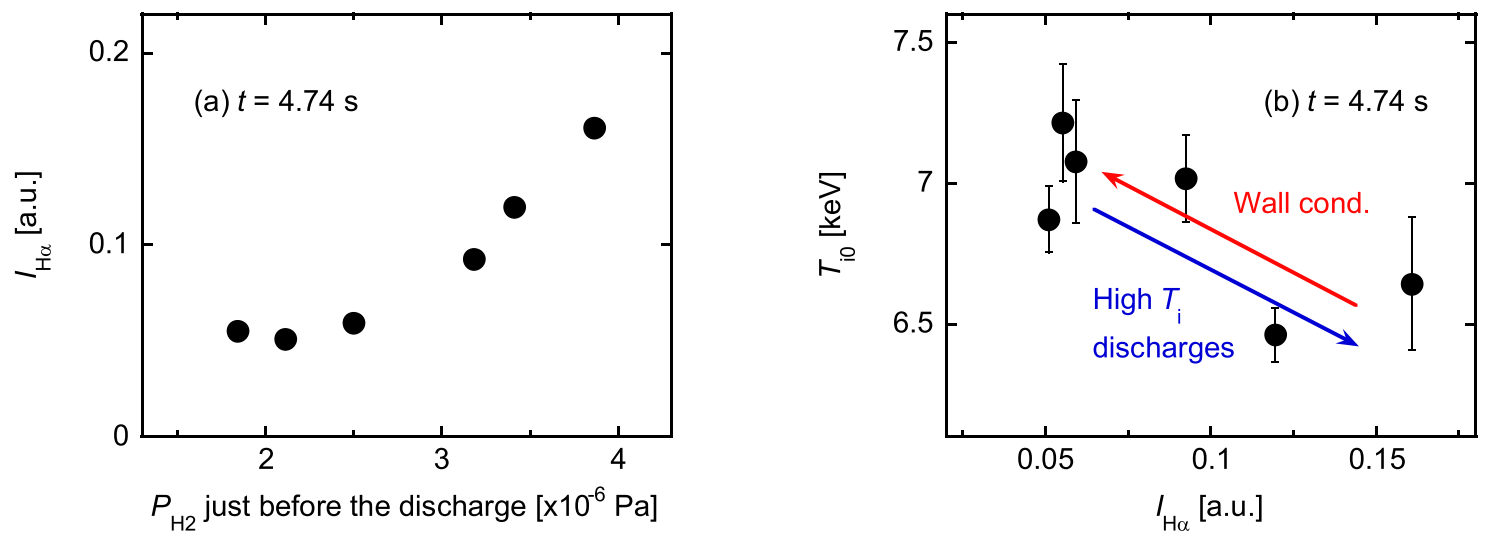

Fig. 4 Dependence of (a) $I_{\mathrm{H} \alpha}$ on $P_{\mathrm{H} 2}$ just before the discharge and (b) $T_{\mathrm{i} 0}$ on $I_{\mathrm{H} \alpha}$ for the six high- $T_{\mathrm{i}}$ discharges in Fig. 3 (b).

LHD experiment day, and (c) the relation between $\Sigma W_{\text {ICRF }}$ and $P_{\mathrm{H} 2}$ just before the next ICRF discharge. The horizontal axis in Fig. 3 (b) is an expansion of that in Fig. 3 (a) and represents the corresponding shot number. The $W_{\text {ICRF }}$ value is the product of the ICRF heating power and the pulse length. The ICRF wall-conditioning discharges were conducted in the hatched period. As seen in Figs. 3 (a) and 3 (b), although plasma with $T_{\mathrm{i} 0}>7 \mathrm{keV}$ could be produced just after the ICRF wall conditioning, the residual hydrogen pressure inside the LHD vacuum vessel gradually increased (please see the bottom envelope of Fig. 3 (a)) as the high- $T_{\mathrm{i}}$ discharges continued and the attained $T_{\mathrm{i} 0}$ decreased. Then, several tens of ICRF wall-conditioning discharges were conducted and $T_{\mathrm{i} 0}$ exceeding $7 \mathrm{keV}$ was obtained again. During the ICRF wall conditioning, the residual hydrogen pressure increased in the initial discharges up to a $\Sigma W_{\text {ICRF }}$ of $50 \mathrm{MJ}$ and then decreased with increasing $\Sigma W_{\text {ICRF }}$ (Fig. 3 (c)). This is evidence that the hydrogen gas stored inside the vacuum vessel components sputtered out because of the ICRF-He plasma.

Figure 4 shows the dependence of (a) $I_{\mathrm{H} \alpha}$ on $P_{\mathrm{H} 2}$ just before the discharge and (b) $T_{\mathrm{i} 0}$ on $I_{\mathrm{H} \alpha}$ for the six high- $T_{\mathrm{i}}$ discharges shown in Fig. 3 (b). The $\mathrm{H} \alpha$ emission decreased with decreasing residual hydrogen pressure before the discharge and the attained $T_{\mathrm{i} 0}$ increased with decreasing $\mathrm{H} \alpha$ emission.

The above results suggest that (1) wall recycling clearly decreased owing to the hydrogen sputtered from the wall because of the ICRF-He discharges, and (2) lowrecycling conditions are critical for realizing high- $T_{\mathrm{i}}$ plasmas.

Similar favorable effects of ECRH instead of ICRF wall-conditioning for realizing high- $T_{\mathrm{i}}$ plasma were observed in the last LHD experimental campaign. In the experiments, several tens of ECRH discharges using $\mathrm{He}$ gas were conducted ( $\left.\Sigma W_{\mathrm{ECRH}} \sim 300 \mathrm{MJ}\right)$ before the high$T_{\mathrm{i}}$ discharges, reduction in the $\mathrm{H} \alpha$ emission, and high $T_{\mathrm{i}}$ were observed. These results imply that not only the highenergy particle flux produced by ICRF but also the total input energy of the RF wave is important for low wallrecycling conditions.

Recently, an experiment to investigate the ICRF-He plasma effect on the microscopic characteristics of the vacuum vessel component was conducted. In this experiment, 
small specimens composed of graphite and stainless steel (SUS316), which are the materials of the diverter and the first wall of the LHD, respectively, were set at the first wall position using a material probe system [16] and were irradiated with a series of ICRF-He plasmas. The experiment will help clarify how the ICRF wall conditioning and the produced high-energy particles affect the hydrogen sputtering characteristics, microscopic modification of the surface, and interior atomic structure of the vacuum vessel components.

\section{Summary}

A new effective wall-conditioning technique was established in the LHD for high- $T_{\mathrm{i}}$ plasmas using the ICRF wave under the established magnetic confinement field. After several repetitive wall-conditioning discharges, we observed a decrease in the line-averaged $n_{\mathrm{e}}$, the formation of a peaked $n_{\mathrm{e}}$ profile, a reduction in the $\mathrm{H} \alpha$ emission, and an increase in the central $T_{\mathrm{i}}$. The results suggest that the stored hydrogen inside the vacuum vessel structures sputtered out because of the He plasmas of the ICRF wall-conditioning discharges and hydrogen recycling decreased. Consequently, the ion heating power of the NBI increased in the plasma core region, leading to high central $T_{\mathrm{i}}$ [11]. By comparing the attained $T_{\mathrm{i} 0}$ in the discharges, just after the different wall-conditioning methods, we find that the ICRF wall conditioning in the established magnetic field is more effective for realizing high- $T_{\mathrm{i}}$ plasmas than the conventional wall-conditioning technique in the
LHD, i.e., He glow discharge and titanium gettering. The effect of the ICRF wall-conditioning discharges on the microscopic modification of the vacuum vessel components will be presented in the near future.

\section{Acknowledgments}

The authors thank Dr. M. Tokitani for fruitful discussions with regard to the PWI characteristics. This work was supported by NIFS grants 11ULRR701, 11ULRR702, and ULRR703.

[1] A. Sagara et al., J. Plasma Fusion Res. 39, 1245 (1999).

[2] D. Tafalla and F.L. Tabares, J. Nucl. Mater. 290-293, 1195 (2001).

[3] K. Nishimura et al., J. Plasma Fusion Res. 79, 1216 (2003).

[4] K. Nishimura et al., J. Nucl. Mater. 337-339, 431 (2005).

[5] E. De Lacal and E. Gauthier, Plasma Phys. Control. Fusion 47, 197 (2005).

[6] V. Rohde et al., J. Nucl. Mater. 363-365, 1369 (2007).

[7] S. Masuzaki et al., Fusion Sci. Technol. 58, 297 (2010).

[8] N. Ohyabu et al., Phys. Rev. Lett. 97, 055002 (2006).

[9] A. Komori et al., J. Nucl. Mater. 390-391, 232 (2009).

[10] K. Nagaoka et al., Nucl. Fusion 51, 083022 (2011).

[11] H. Takahashi et al., Nucl. Fusion 53, 073034 (2013).

[12] H. Yamada et al., Fusion Sci. Technol. 58, 12 (2010).

[13] H. Yamada for the LHD Experiment Group, Nucl. Fusion 51, 094021 (2011).

[14] O. Kaneko et al., Nucl. Fusion 53, 104015 (2013).

[15] S. Morita et al., J. Plasma Fusion Res. 3, S1037 (2008).

[16] M. Tokitani et al., Nucl. Fusion 45, 1544 (2005). 